\title{
INCLUSIÓN DE PERSONAS CON DISCAPACIDADES AUDITIVAS Y VISUALES EN LA INVESTIGACIÓN
}

Inclusion of people with auditive and visual disabilities in research

\author{
Dalia Sánchez*; Rosalinda Romero** y José Padrón***
}

\section{RESUMEN}

Este artículo presenta una estructura teórica en la cual se propone un primer acercamiento a un modelo para la inclusión de las personas con discapacidades auditivas y visuales en la formación de investigadores, mediante el diseño universal, las adaptaciones curriculares y el uso del documento individual de adaptación curricular (DIAC). La fundamentación epistemológica se corresponde con el enfoque deductivista-racionalista y la secuencia procedimental se desarrolló a partir de la observación, delimitación de la estructura empírica, análisis del problema, estudio de las teorías, formulación de hipótesis, construcción del modelo teórico, validación lógica-formal y la derivación de un modelo para la ejecución de las adaptaciones que correspondan, las cuales se fundamentan en las realidades sociales y particulares del contexto, así como el planteamiento de la educación inclusiva como concepción y propósito. Autores como García (2004), Duk y Loren (2010), Rodríguez et al (2012), Feliz y Ricoy (2002) y Booth y Ainscow (2011) fundamentan teóricamente este artículo. El modelo resultante del proceso deductivo propone atender la formación para investigadores desde el enfoque por competencias, la educación inclusiva así como la igualdad y equiparación de oportunidades inherentes a ella, de modo que sea factible que aquellas personas con discapacidades visual y auditiva puedan participar activamente en la investigación.

Palabras clave: Adaptaciones curriculares, diseño universal, discapacidad, formación de investigadores, educación inclusiva.

\footnotetext{
* Personal docente y de investigación. Coordinadora de la Subcomisión de Igualdad y Equiparación de Oportunidades para las Personas con Discapacidad de la Facultad de Arquitectura y Diseño de la Universidad del Zulia (Maracaibo, Venezuela). Correo electrónico: daliasanchez@gmail.com

${ }^{* *}$ Profesora jubilada de la Facultad Experimental de Ciencias de la Universidad del Zulia (Maracaibo, Venezuela). Correo electrónico: rosalindaromerog@gmail.com

*** Profesor jubilado de la Universidad Nacional Abierta (Caracas, Venezuela), Profesor Invitado de LUZ (Maracaibo, Venezuela), Director del Seminario de Epistemología del Posdoctorado en Investigación Cualitativa del INICC (Lima, Perú), Director del Seminario de Investigación del Doctorado de la Universidad de la Amazonia, (Florencia, Colombia). Correo electrónico: josepadrong@gmail.com
} 


\title{
RECIBIDO: Octubre 2018 ACEPTADO: Noviembre 2018
}

\begin{abstract}
This article presents a theoretical structure in which is proposed a first approach to a model for the inclusion of people with hearing and visual disabilities in the training of researchers, through universal design, curricular adaptations and the use of the individual document of curricular adaptation. The epistemological foundation corresponds to the deductivist-rationalist approach and the procedural sequence was developed from the observation, delimitation of the empirical structure, analysis of the problem, study of the theories, formulation of hypotheses, construction of the theoretical model, logical validation- and the derivation of guidelines for the execution of the corresponding adaptations, which are based on the social and particular realities of the context, as well as the approach of inclusive education as conception and purpose. Authors such as García (2004), Duk and Loren (2010), Rodríguez et al (2012), Feliz and Ricoy (2002), Booth and Ainscow (2011) theoretically base this article. The model resulting from the deductive process proposes the training for researchers from the competency-based approach, inclusive education as well as the equality and equalization of opportunities inherent to it, so that it is feasible that those with visual and auditory disabilities can participate actively in the investigation.
\end{abstract}

Key words: Curricular adaptations, disability, universal design, researcher formation, inclusive education

\section{Introducción}

El mundo heterogéneo y diverso demanda respuestas en todos los niveles para garantizar la equiparación de oportunidades. En este sentido, el diseño universal aplicado a la investigación de la mano de ciertos ajustes individuales o adaptaciones curriculares forma parte de las medidas para lograr la educación inclusiva.

Se trata de un proceso complejo de toma de decisiones por parte de las instituciones educativas y sus docentes para la aplicación de estrategias, con el propósito de trabajar en función de las fortalezas del alumnado por encima de las dificultades que pueda experimentar como producto de su relación con el entorno.

Sobre la educación y el específico caso de la investigación, la Convención sobre los Derechos de las Personas con Discapacidad (ONU, 2006), resalta entre sus principios generales la participación e inclusión plenas y efectivas en todas las actividades de la vida, de esta manera y siendo la investigación científica una de las funciones determinantes en el desarrollo social, será clave considerar esos principios a la hora de planificar las acciones inmersas en la formación de investigadores.

Sin embargo, la existencia de instrumentos legales no es suficiente para coordinar la red de intereses organizacionales e individuales que componen cualquier 


\section{Dalia Sánchez; Rosalinda Romero y José Padrón \\ Telos Vol. 21, No. 1 (2019). 221-241}

investigación, se requiere de una visión compartida de los actores en el cómo resolver problemas para incidir en el desarrollo social, sin olvidar que en el caso de las personas con discapacidad serán determinantes la presencia de valores inclusivos arraigados en la institución.

No obstante, y pese a este papel protagónico de la ciencia en el desarrollo, América Latina sigue teniendo resultados muy pobres en lo que respecta a la investigación e innovación. De esta manera, este escenario surge en gran medida de la desarticulación entre las necesidades del entorno y la gerencia de las organizaciones investigativas, las cuales deben tener como norte el desarrollo de conocimientos científicos que den respuestas pertinentes a la situaciones problemáticas del contexto, para ello será también fundamental que los actores del hecho investigativo estén en sintonía con los propósitos (educación) y la ruta para lograrlos (currículo).

Cabe destacar que esta convicción será consecuencia de una organización que parta de la diversidad como una característica inherente a la ciencia, por lo cual todas las personas, independientemente de su género, cultura, religión, idioma así como la presencia o no de una discapacidad, deben tener cabida y serán valorados desde la formación de los investigadores pensando en sus potencialidades para participar activamente en el desarrollo de la sociedad.

De acuerdo con lo anterior, la presente investigación tuvo como objetivo proponer un primer acercamiento con respecto a un modelo para la implementación de adaptaciones curriculares de modo que sea posible alcanzar la inclusión de las personas con discapacidades auditivas y visuales en programas para la formación de investigadores.

\section{Método de estudio}

Esta es una investigación explicativa de alcances teóricos bajo un enfoque deductivista-racionalista, para la cual fue necesario explorar, describir, comprender e interrelacionar los fenómenos en estudio bajo el método deductivo, el cual parte de los hechos al problema, sigue su recorrido a las hipótesis, las teorías, el modelo, las comprobaciones y culmina en las aplicaciones (Olivares de Quintero, 2001).

Este trabajo toma como punto de partida un subcomponente empírico relacionado con las observaciones durante el ejercicio de la docencia, en las que se detectaron ciertos patrones en el caso de las personas con discapacidades auditivas y visuales. A partir de los hechos, se procedió a delimitar la estructura empírica base para el estudio a profundidad de la situación problemática planteada; lo cual derivó en un posterior análisis de teorías que permitieron la formulación de premisas. 
Seguidamente, se construyó el modelo considerando las teorías de sustento sobre discapacidad, inclusión, currículo e investigación. Una vez hecho esto, se avanzó mediante el razonamiento hacia una fase de validación lógica-formal del modelo, para finalizar con la derivación de lineamientos para lograr la inclusión de las personas con discapacidad en programas para la formación de investigadores.

El punto de partida al proceso deductivo fueron las siguientes hipótesis o conjeturas: a) las personas con discapacidad, de acuerdo con sus características individuales y su interacción con el contexto pueden diferenciarse del resto al momento de participar en cualquier proceso de formación, lo cual puede constituirse como causa de discriminación; y, b) en la formación de investigadores se debe considerar la diversidad, incluyendo en ese concepto a personas con distintas discapacidades; de otra manera, parte de la población quedará excluida de la participación en el desarrollo social.

\section{Discapacidades auditivas y visuales}

De acuerdo con la Clasificación Internacional del Funcionamiento, de la Discapacidad y de la Salud (CIF) desarrollada por la Organización Mundial de la Salud (OMS), el funcionamiento se relaciona con funciones, estructuras corporales así como también con las actividades y participación. En contraposición, la discapacidad se corresponde con déficits con consecuencias directas en limitaciones de la actividad y restricciones en la participación (OMS, 2001). En ambos casos, la definición estaría incompleta si se deja por fuera a la interacción entre el individuo y sus factores contextuales (factores ambientales y personales). Conviene agregar que esta interacción puede ser positiva en el caso del funcionamiento y negativa en el caso de la discapacidad.

La Organización Mundial de la Salud (OMS, 2001: 68) relaciona a la discapacidad auditiva con la deficiencia de las «...funciones sensoriales relacionadas con la percepción de los sonidos y la discriminación de su localización, tono, volumen y calidad».

La principal dificultad que enfrentan las personas con discapacidad auditiva es el hecho de lograr comunicarse en su contexto, algo que traerá consecuencias en el desarrollo cognoscitivo, emocional y social. Dicho en otras palabras, la interacción y percepción de los estímulos que les rodean es muy diferente al resto, lo que ejerce gran influencia en su forma de participar en cualquier proceso de formación debido a las consecuencias en el cómo pensar y resolver problemas. 


\section{Dalia Sánchez; Rosalinda Romero y José Padrón}

Telos Vol. 21, No. 1 (2019). 221-241

Por su parte, García (2004) explica que cualquier diferencia en el desarrollo cognitivo no está marcado por la pérdida auditiva; sería causado por la falta de experiencias producto de las dificultades para comunicarse eficazmente, es decir, la sordera por sí sola no determina la evolución intelectual de una persona, pues tal y como se explicó anteriormente, la discapacidad no es un concepto individual, sino que depende de la interacción con el entorno.

En el caso de la descripción de la discapacidad visual y las características de las personas con esta condición, la Organización Nacional de Ciegos Españoles (ONCE, 2018) afirma que las personas con ceguera no pueden ver nada o únicamente tienen una ligera percepción de luz, es decir, aunque pueden ser capaces de diferenciar la luz de la oscuridad, no podrán hacer lo propio en lo que respecta a la forma de los objetos.

Por otro lado, y también de acuerdo a lo expuesto por la ONCE (2018) las personas con baja visión son aquellas que con la mejor corrección posible pueden distinguir algunos objetos muy cercanos, aunque con gran dificultad. En algunos casos, y con el suficiente contraste, las personas con baja visión pueden leer con ayudas técnicas, caracteres ampliados o macrotipos, sin embargo es una actividad que hacen lentamente debido al esfuerzo que implica.

Según García (2011) las personas con discapacidad visual tendrán debilidades para la representación mental, debido a que su conocimiento de la realidad parte de estímulos táctiles y auditivos, algo que sin duda será más notable en aquellas personas cuya condición sea congénita o adquirida en edades tempranas.

En este orden de ideas, las personas ciegas o con baja visión se apoyan en la audición, el tacto y el lenguaje para su participación activa en la sociedad, siempre que sea posible determinar la relación entre el sonido y su causa. En otras palabras, la información obtenida a través del lenguaje configura en gran medida los significados, sobre todo aquellos que no puedan ser percibidos a través del tacto.

En lo que respecta al lenguaje escrito, por necesitar mayores capacidades visuales, podrá desarrollarse o no según el nivel de funcionamiento de los sujetos. Tal como explica Salvador (2001) las principales dificultades para la lectoescritura se localizan en los procesos mecánicos como la decodificación de los signos gráficos, en el caso de la lectura, y en la transcripción del texto, en el caso de la escritura.

\section{Educación inclusiva}

Según Blanco (2008: 5) la educación es uno de los caminos hacia la inclusión social y esta será posible a partir del «...desarrollo de escuelas o contextos educativos que acojan a todas las personas de la comunidad, independientemente de su procedencia social, cultural o características individuales, y den respuesta a la diversidad de necesidades de aprendizaje». Es decir, el cambio en las estructuras ha de responder a la 
diversidad de los estudiantes, algo que va más allá de su origen, color de piel o la presencia o no de una discapacidad, resaltan también las diferencias en cómo resolver problemas desde los más cotidianos hasta los más complejos.

Por su parte, Parra (2010: 77) agrega que «La educación inclusiva constituye un enfoque educativo basado en la valoración de la diversidad como elemento enriquecedor del proceso de enseñanza y aprendizaje y, en consecuencia, favorecedor del desarrollo humano».

Booth \& Ainscow (2011: 25) afirman que la inclusión como proceso es sinónimo de compromiso por promover la participación y alertan sobre el peligro de que no esté acompañada de valores arraigados, pues «...puede representar simplemente la conformidad con una "nueva moda educativa" o la complacencia superficial con las instrucciones que emanan de la administración».

Como consecuencia de estas preocupaciones, y con el norte de apoyar los procesos de transformación hacia una educación inclusiva, los mencionados autores propusieron en el año 2000 el denominado índice inclusión, que incluye procesos de exploración, análisis, desarrollo de planes, implementación de acción y una permanente revisión del proceso a seguir. Ahora bien, aunque el Índice de Inclusión fue inicialmente diseñado para el ámbito escolar, sus dimensiones son aplicables en todos los niveles de cualquier sistema.

Según Booth \& Ainscow (2011) hay tres dimensiones interconectadas esenciales para lograr cambios positivos y ellas son: i) Creando culturas inclusivas; ii) Estableciendo políticas inclusivas; y, iii) Desarrollando prácticas inclusivas. Se trata de una relación incluyente de mayor a menor, dicho en otras palabras el aspecto más macro corresponde a la CULTURA, la cual incluye a las POLÍTICAS y estas a su vez a las PRÁCTICAS INCLUSIVAS. Para ilustrar lo anteriormente expuesto se presenta el siguiente gráfico.



Gráfico 1 Dimensiones de la inclusión 


\section{Dalia Sánchez; Rosalinda Romero y José Padrón \\ Telos Vol. 21, No. 1 (2019). 221-241}

Fuente: Basado en Booth \& Ainscow (2011, Pág. 49)

En primera instancia y de acuerdo con Booth \& Ainscow (2011) el crear culturas inclusivas se relaciona con el hecho de que todos los miembros de la comunidad aceptan y valoran la diversidad. De esta manera, una cultura inclusiva como forma de vida guiará el desarrollo de políticas y en la práctica del día a día, lo que significa también que estos valores pueden irse transmitiendo a los nuevos integrantes.

En segunda instancia, la dimensión referida al establecimiento de políticas inclusivas busca aplicar los valores de la cultura para reforzar la participación de todos, lo cual será posible siempre que se tenga la capacidad de atender la diversidad, valorar con equidad y romper barreras. Mientras que en tercera instancia, el desarrollar prácticas inclusivas implica reflexionar directamente sobre la enseñanza y el aprendizaje en las aulas.

Estas tres instancias descritas implican no solamente la aceptación de la diversidad, sino un proceso permanente de reflexión sobre las barreras que puedan existir y las formas de romperlas.

\section{Adaptaciones curriculares}

Grau y Fernández (2008) definen a las adaptaciones curriculares como un proceso en el cual se toman decisiones sobre los elementos que constituyen el currículo, así como también en lo referido a aquellos factores determinantes en el acceso al mismo por parte de las personas con discapacidad. Las mismas autoras afirman que el término de adaptación curricular, entendido como adaptación de la enseñanza, busca satisfacer necesidades de la diversidad de alumnos y solo será factible cuando provengan de un currículo común y flexible.

Surge entonces la necesidad de considerar la aplicación del diseño universal aplicado a la educación en el desarrollo de cualquier currículo que tenga la cualidad de flexible, puesto que tal como explica Bromberg (2004) se trata de un proceso relacionado con la permanente resolución de problemas cuyo fin no es un objeto, sino que se centra en la persona.

De este modo, y como respuesta a la problemática expuesta, los principios del diseño universal buscan simplificar la vida con el desarrollo de productos, comunicaciones y ambientes de fácil acceso para la mayor cantidad de personas posibles y al menor costo, para ello resalta la importancia de que esta meta se logre sin necesidad de adaptaciones o diseños especiales (Mace, 1997). 
En este tenor, es importante resaltar que el diseño universal sigue siendo un campo de estudio y reflexión que está alejado de parámetros y especificaciones técnicas, y que ha servido de base para diversas iniciativas educativas las cuales coinciden en buscar la participación de todos los estudiantes sin distinción.

Entre estas iniciativas cabe mencionar al Diseño Universal para la Instrucción (DUI), el cual es una propuesta de Palmer \& Caputo (2002) que fue traducida y adaptada por un equipo de expertos de las Universidades Ramón Llull y Politécnica de Cataluña. Este material no solamente explica cada principio, sino que incluye preguntas de reflexión sobre el método de enseñanza y aprendizaje, ejemplos con buenas prácticas y unos apéndices con recomendaciones de acción a corto, mediano y largo plazo

Por otro lado, la toma de decisiones en lo que respecta a la práctica en el aula forma parte del día a día del docente de todos los niveles en mayor o menor medida, y esta dependerá de la diversidad de respuestas y de necesidades de los individuos en el transcurso de la enseñanza-aprendizaje e implica un trabajo que debe partir de un estudio completo del estudiante en el entorno.

Hecha esta observación, Duk y Loren (2010) explican los principios básicos para la implementación de adaptaciones curriculares, los cuales se resumen a continuación: a) El proceso de adaptación del currículo y sus acciones deben estar en el proyecto institucional; b) Las adaptaciones curriculares deben fundamentarse en una evaluación amplia y rigurosa de los estudiantes en interacción con el contexto; c) Debe considerarse la relevancia y pertinencia para el presente y futuro del estudiante; d) Deben estar ligadas a la programación inicial; e) Deben ser el resultado de un trabajo interdisciplinario y colaborativo; f) Deben estar sujetas a mecanismos de seguimiento, regulación y control.

En lo que respecta a la clasificación de las adaptaciones curriculares, diversidad de autores explican que pueden ser de acceso e individualizadas. Las adaptaciones de acceso surgen como respuesta a aquellos factores que por acción u omisión en aspectos físicos, materiales y de comunicación hacen difícil la participación de las personas con discapacidad.

Rodríguez et al (2012) explican que las adaptaciones de aspectos físicos deben favorecer la autonomía personal de todos los individuos mediante la supresión de las diferentes barreras arquitectónicas relacionadas con la disposición del mobiliario, iluminación, sonido, entre otras. Del mismo modo, al momento de desarrollar materiales educativos será positivo que sea atractivo tanto para el destinatario con discapacidad, como para el resto de sus compañeros.

En otro orden de ideas, la última gran dificultad que enfrentan muchos estudiantes y por ende investigadores con discapacidad viene determinada por 


\section{Dalia Sánchez; Rosalinda Romero y José Padrón \\ Telos Vol. 21, No. 1 (2019). 221-241}

interferencias dentro del proceso de comunicación, las cuales pueden iniciar desde el registro o inscripción en cualquier plan de formación.

Resulta oportuno aclarar que las posibles interferencias no significan necesariamente que las personas no cuentan con la capacidad de aprender, sino que las vías de comunicación empleadas no se corresponden con las características individuales del sujeto.

Así, según la opinión de quienes suscriben, el docente como mediador del conocimiento y en concordancia con lo dicho en apartados anteriores, deberá reflexionar sobre los medios de interacción disponibles, así como sus ventajas y desventajas para obtener su máximo provecho, en todos los procesos inherentes a la educación, siendo la evaluación uno de los que puede generar mayor debate entre los docentes.

La búsqueda entonces debe estar orientada a técnicas que permitan la evaluación justa de los aprendizajes de aquellos con discapacidad, para con ello garantizar el éxito del proceso enseñanza-aprendizaje.

En resumidas cuentas y tal como lo exponen Rodríguez et al (2012) serán las características específicas de los estudiantes los referentes principales para la gestión de los recursos. Sobre este particular, los autores hacen énfasis en los recursos humanos tales como intérpretes de lengua de señas y acciones tutoriales de los docentes.

De esta manera, cualquier centro de formación debe garantizar el cumplimiento de normas de accesibilidad en los recursos materiales educativos, lo cual debe repetirse en cualquier emisión de mensajes institucionales para públicos externo e interno, de modo que permitan llegar a todas las personas sin barreras.

En lo que respecta a las adaptaciones curriculares individualizadas, se trata de acciones centradas en la satisfacción de las necesidades educativas del estudiante, en este caso investigador. En un primer nivel de adaptaciones curriculares individualizadas se encuentran las no significativas, las cuales no implican grandes cambios, sino ajustes según los requerimientos de los estudiantes.

Según Rodríguez et al (2012: 93) se recomiendan las adaptaciones no significativas cuando el desfase es poco notable y afecta, a pocos elementos del currículo por lo cual no se modifican sustancialmente objetivos o métodos de evaluación.

En contraposición, las adaptaciones significativas sugieren cambios más drásticos en los currículos con consecuencias en los contenidos, objetivos y formas de evaluación, lo que se traduce en una fuerte variación de las habilidades a adquirir. También entrarán en esta categoría los cambios referidos a los procedimientos o instrumentos de evaluación. 
En este sentido, Rodríguez et al (2012: 94) recomiendan que este tipo de adaptaciones sean diseñadas por especialistas, junto al profesorado del área correspondiente.

Para finalizar, las adaptaciones curriculares muy significativas son medidas tan extraordinarias que se alejan de la práctica inclusiva pues se relacionan con eliminaciones de contenidos u objetivos educativos.

A partir de las consideraciones anteriores sobre discapacidad, diseño universal y adaptaciones curriculares, conviene anexar una esquematización de las principales dificultades que experimentan las personas con discapacidad sensorial junto a sus alternativas de solución.

Las siguientes son aproximaciones generales, puesto que para poder hacer una propuesta exacta será necesario conocer las características del sujeto, además de los recursos disponibles y medidas factibles de aplicar.

Cuadro 1. Dificultades y soluciones para personas con discapacidad visual

\begin{tabular}{|l|l|}
\hline \multicolumn{2}{|c|}{ Baja visión/Ceguera total } \\
\hline Dificultades & Soluciones \\
\hline $\begin{array}{l}\text { 1. Restricciones para la representación } \\
\text { mental de términos abstractos con gran } \\
\text { influencia visual }\end{array}$ & $\begin{array}{l}\text { 1. Verbalizar o describir todos los términos o } \\
\text { conceptos }\end{array}$ \\
\hline $\begin{array}{l}\text { 2. Restricciones para la representación por } \\
\text { el poco conocimiento de la realidad }\end{array}$ & $\begin{array}{l}\text { 2. Verbalizar o describir todos los términos o } \\
\text { conceptos }\end{array}$ \\
\hline $\begin{array}{l}\text { 3. La contaminación acústica produce } \\
\text { pérdidas de información auditiva }\end{array}$ & $\begin{array}{l}\text { 3. Acústica controlada } \\
\text { Instaurar orden de intervenciones }\end{array}$ \\
\hline $\begin{array}{l}\text { 4. Más lento que el promedio para lectura } \\
\text { y escritura }\end{array}$ & $\begin{array}{l}\text { 4. Más tiempo para lectura y escritura } \\
\text { Facilitar con antelación el material } \\
\text { bibliográfico en el formato que requiera el } \\
\text { estudiante }\end{array}$ \\
\hline
\end{tabular}

Fuente: Sánchez (2017)

Cuadro 2. Dificultades y soluciones para personas con discapacidad auditiva

\begin{tabular}{|l|l|l|l|}
\hline \multicolumn{4}{|c|}{ Discapacidad Auditiva } \\
\hline Hipoacusia & Soluciones & Sordera profunda \\
\hline Dificultades & Dificultades & Soluciones \\
\hline 1. Limitaciones en & - Buscar una manera & 1. Sin competencias & - Buscar una manera \\
el lenguaje oral & de comunicación & comunicativas orales & de comunicación \\
& funcional & & funcional \\
& - Apoyarse en la & & - Apoyarse en la \\
& lectura labiofacial. & & \\
\hline
\end{tabular}




\begin{tabular}{|c|c|c|c|}
\hline & $\begin{array}{l}\text { - Recurrir a la } \\
\text { interpretación en } \\
\text { lengua de señas } \\
\text { cuando corresponda }\end{array}$ & & $\begin{array}{l}\text { - Recurrir a la } \\
\text { interpretación en } \\
\text { lengua de señas }\end{array}$ \\
\hline $\begin{array}{l}\text { 2. Restricciones } \\
\text { para la formulación } \\
\text { de hipótesis }\end{array}$ & $\begin{array}{l}\text { - Compensación de } \\
\text { tiempo } \\
\text { - Uso de recursos } \\
\text { visuales, mapas } \\
\text { conceptuales }\end{array}$ & $\begin{array}{l}\text { 2. Grandes } \\
\text { restricciones para la } \\
\text { formulación de } \\
\text { hipótesis }\end{array}$ & $\begin{array}{l}\text { - Compensación de } \\
\text { tiempo } \\
\text { - Uso de recursos } \\
\text { visuales, mapas } \\
\text { conceptuales } \\
\text { - Recurrir a la } \\
\text { interpretación en } \\
\text { lengua de señas }\end{array}$ \\
\hline $\begin{array}{l}\text { 3. Restricciones en } \\
\text { actividades lógicas } \\
\text { para la abstracción }\end{array}$ & $\begin{array}{l}\text { - Compensación de } \\
\text { tiempo } \\
\text { - Uso de recursos } \\
\text { visuales, mapas } \\
\text { conceptuales } \\
\text { - Evaluar además del } \\
\text { producto final el } \\
\text { proceso }\end{array}$ & $\begin{array}{l}\text { 3. Grandes } \\
\text { restricciones en } \\
\text { actividades lógicas } \\
\text { para la abstracción }\end{array}$ & $\begin{array}{l}\text { - Compensación de } \\
\text { tiempo } \\
\text { - Uso de recursos } \\
\text { visuales, mapas } \\
\text { conceptuales } \\
\text { - Evaluar además del } \\
\text { producto final el } \\
\text { proceso } \\
\text { - Recurrir a la } \\
\text { interpretación en } \\
\text { lengua de señas }\end{array}$ \\
\hline
\end{tabular}

Fuente: Sánchez (2017)

Cuadro 3. Dificultades y soluciones para personas con discapacidad auditiva (continuación)

\begin{tabular}{|c|c|c|c|}
\hline \multicolumn{4}{|c|}{ Discapacidad Auditiva } \\
\hline \multicolumn{2}{|l|}{ Hipoacusia } & \multicolumn{2}{|l|}{ Sordera profunda } \\
\hline Dificultades & Soluciones & Dificultades & Soluciones \\
\hline $\begin{array}{l}\text { 4. Restricciones } \\
\text { para la comprensión } \\
\text { de estructuras no } \\
\text { observables, } \\
\text { contenido figurativo } \\
\text { y metafórico }\end{array}$ & $\begin{array}{l}\text { - Compensación de } \\
\text { tiempo } \\
\text { - Uso de recursos } \\
\text { visuales, mapas } \\
\text { conceptuales } \\
\text { - Evaluar además } \\
\text { del producto final el } \\
\text { proceso }\end{array}$ & $\begin{array}{l}\text { 4. Grandes } \\
\text { restricciones para la } \\
\text { comprensión de } \\
\text { estructuras no } \\
\text { observables, } \\
\text { contenido figurativo } \\
\text { y metafórico }\end{array}$ & $\begin{array}{l}\text { - Compensación de } \\
\text { tiempo } \\
\text { - Uso de recursos } \\
\text { visuales, mapas } \\
\text { conceptuales } \\
\text { - Evaluar además } \\
\text { del producto final el } \\
\text { proceso } \\
\text { - Recurrir a la } \\
\text { interpretación en } \\
\text { lengua de señas }\end{array}$ \\
\hline $\begin{array}{l}\text { 5. Restricciones en } \\
\text { la interacción con } \\
\text { oyentes en }\end{array}$ & $\begin{array}{l}\text { - Ordenar turnos de } \\
\text { palabra, si es posible } \\
\text { incluir un objeto o } \\
\text { testigo en la }\end{array}$ & $\begin{array}{l}\text { 5. Aislamiento del } \\
\text { entorno oyente }\end{array}$ & $\begin{array}{l}\text { - Ordenar turnos de } \\
\text { palabra, si es posible } \\
\text { incluir un objeto o } \\
\text { testigo en la }\end{array}$ \\
\hline
\end{tabular}




\begin{tabular}{|c|c|c|c|}
\hline $\begin{array}{l}\text { plenarias, debates y } \\
\text { otros }\end{array}$ & $\begin{array}{l}\text { dinámica para } \\
\text { marcar el turno, etc. } \\
\text { - Recurrir a una } \\
\text { pizarra a manera de } \\
\text { registro con palabras } \\
\text { claves } \\
\text { - Recurrir a la } \\
\text { interpretación en } \\
\text { lengua de señas } \\
\text { cuando corresponda }\end{array}$ & & $\begin{array}{l}\text { dinámica para } \\
\text { marcar el turno, etc. } \\
\text { - Recurrir a una } \\
\text { pizarra a manera de } \\
\text { registro con palabras } \\
\text { claves } \\
\text { - Recurrir a la } \\
\text { interpretación en } \\
\text { lengua de señas } \\
\text { cuando corresponda }\end{array}$ \\
\hline $\begin{array}{l}\text { 6. Restricciones en } \\
\text { el vocabulario, tanto } \\
\text { en comprensión } \\
\text { como en expresión } \\
\text { hablada y escrita. } \\
\text { Interpretación de } \\
\text { textos de forma } \\
\text { literal }\end{array}$ & $\begin{array}{l}\text { - Facilitar } \\
\text { información } \\
\text { específica en forma } \\
\text { esquemática o } \\
\text { resumida } \\
\text { - Usar sinónimos y } \\
\text { conceptos asociados } \\
\text { - Recurrir a la } \\
\text { interpretación en } \\
\text { lengua de señas } \\
\text { cuando corresponda }\end{array}$ & $\begin{array}{l}\text { 6. Escaso } \\
\text { vocabulario, tanto } \\
\text { en comprensión } \\
\text { como en expresión } \\
\text { hablada y escrita. }\end{array}$ & $\begin{array}{l}\text { - Facilitar } \\
\text { información } \\
\text { específica en forma } \\
\text { esquemática } \\
\text { - Recurrir a la } \\
\text { interpretación en } \\
\text { lengua de señas } \\
\text { cuando corresponda }\end{array}$ \\
\hline
\end{tabular}

Fuente: Sánchez (2017)

\section{Documento Individual de Adaptación Curricular (DIAC)}

Rodríguez et al (2012) explican la importancia de redactar un Documento Individual de Adaptación Curricular (DIAC) el cual debe partir de un informe psicopedagógico del estudiante, ya que a diferencia del caso de las adaptaciones no significativas, hay un desfase curricular notable que exige modificaciones de forma individual en elementos fundamentales del currículo. Sin embargo, puede considerarse que el DIAC será un instrumento valioso para hacer el seguimiento del aprendizaje y rendimiento de todo el alumnado con discapacidad, independientemente del tipo de adaptación curricular que requiera para llenar los posibles vacíos dejados por el diseño universal aplicado a la educación.

Por su parte, Feliz y Ricoy (2002) proponen un proceso para la implementación del DIAC compuesto por cinco fases que se basa en el publicado por el Centro de Investigación y Documentación Educativa por Martín (1999) y ellas son: a) recogida de información, b) verificación de las necesidades; c) propuesta de adaptación; d) medidas necesarias para la implementación; y, e) elementos operativos de apoyo y evaluación de la adaptación curricular 
Los mencionados autores consideran que la recolección de información debe ser profunda y selectiva al mismo tiempo, pues no todos los datos son igualmente importantes para ser considerados en el proceso de implementación.

A juicio de los investigadores será de suma relevancia el tener a disposición una evaluación psicológica actualizada, la cual junto al informe médico correspondiente será una guía fundamental para que los docentes puedan tener una visión objetiva de las potencialidades y dificultades de los investigadores en formación.

La sistematización del proceso que da cabida a los éxitos y fracasos constituye una muestra de la evolución de los sujetos en los planes de formación y marcan el camino de las estrategias que más funcionan al momento de enfrentar los retos de casos similares.

De esta manera, serán relevantes los insumos generados de la observación, los productos o evidencias de las evaluaciones, así como también entrevistas o reuniones con todos los actores del hecho educativo.

Ahora bien, como en cualquier instrumento de trabajo se deben incluir datos objetivos que deben ser usados de manera confidencial por los docentes con fines académicos. Estos datos son: a) nombres y apellidos, fecha y lugar de nacimiento, vías de contacto y modalidades de comunicación adecuadas; b) situación académica; c) discapacidad o condición, tipo, forma y el momento de la aparición, así como también las principales consecuencias en su funcionamiento; d) Uso de dispositivos técnicos y/o tecnologías de apoyo; y, e) historial, adaptaciones curriculares anteriores, tipos de actividades más efectivas, recursos y ayudas pedagógicas más favorables, métodos más pertinentes, rendimiento y formas de evaluación con mejores resultados.

\section{Gráfico 2. Primera parte del DIAC}


Inclusión de personas con discapacidades auditivas y visuales en la investigación

\begin{tabular}{|c|c|c|c|}
\hline \multicolumn{4}{|c|}{ Documento Individual de Adaptación Curricular (DIAC) } \\
\hline \multicolumn{4}{|l|}{ Identificación } \\
\hline Nombre: & & Apellido: & \\
\hline $\begin{array}{l}\text { Documento de } \\
\text { identidad: }\end{array}$ & & Dirección: & \\
\hline Fecha de nacimiento: & & $\begin{array}{l}\text { Lugar de } \\
\text { nacimiento: }\end{array}$ & \\
\hline \multicolumn{4}{|l|}{ Vías de contacto } \\
\hline Teléfono: & & Correo electrónico & \\
\hline Usuario en Twitter: & & $\begin{array}{l}\text { Usuario en } \\
\text { Facebook }\end{array}$ & \\
\hline Usuario en Instagram & & Otras redes & \\
\hline \multicolumn{4}{|l|}{ Situación académica } \\
\hline \multicolumn{4}{|l|}{ Semestre/Año } \\
\hline $\begin{array}{l}\text { Adaptaciones } \\
\text { curriculares previas }\end{array}$ & Sí( ) No( ) & ¿Cuáles? & \\
\hline $\begin{array}{l}\text { Unidades curriculares/ } \\
\text { seminarios } \\
\text { reprobados }\end{array}$ & Sí( ) No( ) & ¿Cuáles? & \\
\hline \multicolumn{4}{|l|}{ Discapacidad } \\
\hline Tipo & & Nivel: & \\
\hline Congénita & Sí( ) No ( ) & Adquirida & Sí( ) No ( ) \\
\hline $\begin{array}{l}\text { Dispositivos } \\
\text { técnicos y/o } \\
\text { tecnologías de } \\
\text { apoyo }\end{array}$ & Sí( ) No( ) & ¿Cuáles? & \\
\hline \multicolumn{2}{|c|}{ Consecuencias en el funcionamiento: } & \multicolumn{2}{|c|}{$\begin{array}{l}\text { Dificultades en el proceso de } \\
\text { aprendizaje: }\end{array}$} \\
\hline Observaciones: & & & \\
\hline
\end{tabular}

Fuente: Sánchez (2017)

En la misma dirección, también conviene tener un registro de iniciativas, motivaciones, receptividad, temores, capacidad de reflexión, estrategias para lo solución de problemas, reacción ante las dificultades, interacción con el entorno, niveles de autonomía, así como las reacciones ante el éxito o el error.

Tomando como punto de partida la información recabada, se deben contrastar las características descritas con las competencias planteadas. Igualmente, hay que tomar como referencia el desempeño del resto de los compañeros para entender los posibles niveles de desfases y tratar de aplicar las estrategias de acuerdo con las características del estudiante y a las dificultades que experimenta al interactuar en el contexto

$\mathrm{Al}$ tener las posibles soluciones, las mismas pueden ser clasificadas en función de sus características, para que sea más fácil su comprensión dentro del documento. En 


\section{Dalia Sánchez; Rosalinda Romero y José Padrón}

Telos Vol. 21, No. 1 (2019). 221-241

este sentido, los investigadores consideran oportuno recordar que el hecho de lograr soluciones en pro del diseño universal de materiales físicos, digitales o hasta las instalaciones de mobiliario traerá beneficios para todo el entorno y no únicamente para las personas con discapacidad.

Igualmente, como todo proceso, la implementación de las adaptaciones curriculares requiere de un seguimiento individual, de modo que se puedan hacer los ajustes necesarios tomando en cuenta los resultados de la práctica.

Durante el funcionamiento de las estrategias diseñadas para un determinado investigador, se debe realizar un monitoreo permanente que permita, con ayuda del registro, hacer cambios pertinentes en caso de ser necesarios, hasta obtener los resultados esperados. Con este propósito, es importante que todas las personas involucradas en el proceso tengan su voz y reporten cualquier incidencia durante la implementación de las adaptaciones curriculares como la única manera de garantizar mejoras en el proceso.

Ahora bien, y con el propósito de poder incluir, después de la información personal de cada investigador, la determinación de las dificultades y alternativas para cada competencia de acuerdo a la discapacidad y nivel de funcionamiento en el documento individual de adaptación curricular (DIAC), se muestra otro cuadro de trabajo a manera de ejemplo para lograr la implementación de las adaptaciones curriculares.

Gráfico 3. Segunda parte del Documento Individual de Adaptación Curricular (DIAC)

\begin{tabular}{|c|c|c|}
\hline \multicolumn{3}{|c|}{ ASPECTO ESENCIAL PARA LA FORMACION DE INVESTIGADORES } \\
\hline Indicadores conceptuales & $\begin{array}{l}\text { Indicadores } \\
\text { procedimentales }\end{array}$ & \begin{tabular}{|l|} 
Indicadores \\
actitudinales
\end{tabular} \\
\hline C1. XXXX & P1. XXXX & A1. XXXX \\
\hline C2. XXXX & P2. $X X X X$ & A2. $X X X X$ \\
\hline C3. $X X X X$ & P3. $X X X X$ & A3. $X X X X$ \\
\hline Estrategias de evaluación: & Estrategias de evaluación: & $\begin{array}{l}\text { Estrategias de } \\
\text { evaluación: }\end{array}$ \\
\hline$X X X X$ & $X X X X$ & $X X X X$ \\
\hline Dificultades & \multicolumn{2}{|l|}{ Soluciones } \\
\hline Observaciones: & & \\
\hline
\end{tabular}

Fuente: Sánchez (2017)

En dicho cuadro, las adaptaciones se corresponden con los indicadores de los aspectos actitudinales, psicosociológicos, macrosociológicos, psicoafectivos, lógicos metodológicos, discursivos y lingüísticos, así como estratégicos y curriculares descritos 
por Padrón (2002) y otros investigadores de la Línea de Investigaciones en Enseñanza/Aprendizajes de la Investigación (LINEA-I).

Resultará oportuno entonces que los mencionados aspectos esenciales en la formación de investigadores puedan expresarse bajo el enfoque por competencias, tomando como punto de partida que estas se desarrollan según las necesidades del contexto (Inciarte y Canquiz, 2008) con la finalidad de reducir distancias entre la formación académica y el ejercicio profesional (Casanova, 2011). En otras palabras, la formación de investigadores constituye una conexión entre la educación y el desempeño profesional en las organizaciones investigativas.

En consecuencia, las competencias pueden establecerse como metas que al ser analizadas a la luz de las características de los sujetos con discapacidades auditivas y visuales, así como las posibles dificultades específicas asociadas, permitan la toma las previsiones curriculares para el éxito de la formación.

\section{Resultados}

\section{MODELO PARA LA INCLUSIÓN DE LAS PERSONAS CON DISCAPACIDADES AUDITIVAS Y VISUALES EN LA INVESTIGACIÓN}

Antes de esquematizar el escenario ideal para la implementación de las adaptaciones curriculares, como parte de las políticas de apoyo para el alumnado con discapacidad, debe aclararse que en esta propuesta no se consideran situaciones coyunturales como crisis políticas, económicas y sociales de los países, situaciones que sin duda son determinantes para la toma de decisiones en las instituciones y limitan acciones para la intervención en distintos niveles de accesibilidad con facilitadores para el desempeño y la participación plena en la educación.

Igualmente, este escenario considera las dimensiones de la inclusión, planteadas por Booth y Ainscow (2011) y ellas son: A) Creando culturas inclusivas; B) Estableciendo políticas inclusivas; y, C) Desarrollando prácticas inclusivas.

En este contexto caracterizado por valores inclusivos arraigados, se desarrollan políticas para lograr que en los entornos físico, social y actitudinal reinen los facilitadores para la formación, desempeño y participación de las personas con discapacidad en la investigación.

La institución declara dentro de su concepción la educación inclusiva como norte, de modo que para alcanzarla trabaja por una cultura en la cual la igualdad y equiparación de oportunidades son primordiales, esto se traduce en que todos los integrantes de la comunidad tienen sentido de pertinencia y son valorados como iguales. En los elementos que dan forma al macrocurrículo, se consideran los planes nacionales y mundiales para la definición del hombre en una sociedad más justa. 
En este sentido, los programas de las unidades curriculares parten del Diseño Universal aplicado a la educación para las previsiones iniciales. Luego, de acuerdo a un análisis profundo de cada investigador con discapacidad sensorial, se podrá desarrollar un Documento Individual de Adaptación Curricular (DIAC), el cual incluye dificultades específicas, de acuerdo al tipo y grado de discapacidad, así como alternativas en función a las competencias a adquirir, en las cuales están declarados los valores inclusivos en el componente actitudinal. Cabe destacar que para mejorar o perfeccionar el proceso se hacen necesarias medidas de gestión y control que formen parte dicho documento.

El resultado será que con la implementación de las adaptaciones a la medida de las necesidades del plan de formación y potencialidades de los investigadores, la diversidad de investigadores con discapacidad estará eficazmente formada e incluida en la institución y por ende participarán activamente en el desarrollo social a través de la práctica de la investigación.

En este contexto caracterizado por valores inclusivos arraigados, se desarrollan políticas para lograr que en los entornos físico, social y actitudinal reinen los facilitadores para la formación, desempeño y participación en la investigación.

En el siguiente diagrama de flujo se resaltan las principales acciones para lograr su inclusión en las organizaciones investigativas.

Gráfico 4. Inclusión de personas con discapacidad en organizaciones investigativas 
Inclusión de personas con discapacidades auditivas y visuales en la investigación

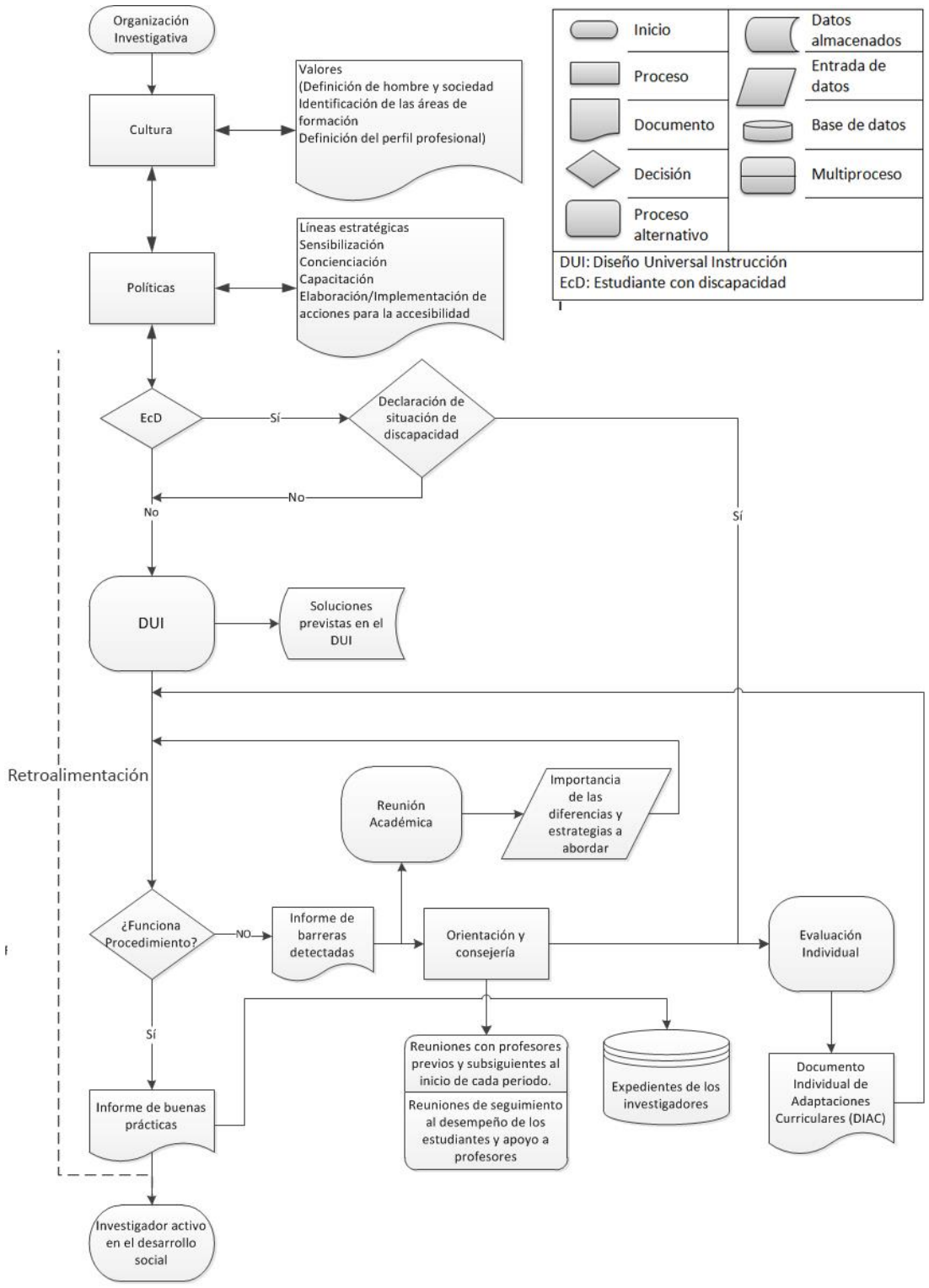

Fuente: Sánchez (2017)

\section{Conclusiones}

De acuerdo con el objetivo planteado, la presente investigación propone un primer acercamiento con respecto a un modelo para la implementación de adaptaciones 


\section{Dalia Sánchez; Rosalinda Romero y José Padrón \\ Telos Vol. 21, No. 1 (2019). 221-241}

curriculares de modo que sea posible alcanzar la inclusión de las personas con discapacidades auditivas y visuales en programas para la formación de investigadores.

En este sentido, se presenta una respuesta conceptual que resalta las interrelaciones entre los factores curriculares y las necesidades específicas por discapacidad, para así alcanzar la formación de todos los investigadores sin discriminación. Se trata entonces de una estructura teórica que para llevarla al terreno de la praxis amerita un trabajo interdisciplinario de modo que los cambios no sean simplemente impuestos, sino que permitan la apreciación de la diversidad de formas para alcanzar el conocimiento científico como un paso clave en la construcción de sociedades más justas.

El hallazgo central, según las pautas del objetivo de trabajo, es la sistematización de la implementación de las adaptaciones curriculares esenciales por niveles, considerando en primer lugar a aquellas generales que implican medidas en la institución en lo referido al acceso, materiales y comunicación; mientras que, en segundo lugar, se parte de la individualidad de cada investigador.

Se puso de manifiesto en el estudio que, la implementación de las adaptaciones curriculares llena los posibles vacíos dejados por el diseño universal aplicado a la educación, puesto que de acuerdo al tipo, momento de aparición de la discapacidad, su nivel y otros; se tiene un amplio espectro de diferencias para lo cual se deben ir ajustando las respuestas según el contexto institucional y curricular.

En este sentido, las interacciones entre los investigadores y el contexto, podrán ser positivas o negativas según puedan garantizarse su participación en los procesos llevados a cabo en la organización investigativa de manera digna con autonomía, normalidad, comodidad y seguridad.

Finalmente, se pudo presentar un escenario ideal de políticas de apoyo a los investigadores con discapacidad para la implementación de adaptaciones curriculares en función de la concepción de educación inclusiva, la existencia de valores inclusivos arraigados, el desarrollo de políticas que valoren las diferencias y prácticas docentes que implemente o no modificaciones en el currículo de acuerdo a las necesidades y potencialidades de los investigadores en formación.

La estructura teórica que se esboza aspira a ser contrastada eventualmente y con ello favorecer a una percepción libre de prejuicios sobre la participación de las personas con discapacidad sensorial en la educación, y más específicamente en la ciencia.

\section{Referencias bibliográficas}

Blanco, Rosa. (2008). Marco conceptual sobre educación inclusiva. La educación inclusiva. El camino hacia el futuro. Conferencia internacional de educación. Unesco. 
http://www.ibe.unesco.org/fileadmin/user_upload/Policy_Dialogue/48th ICE/CONFINTED_48_Inf_2_Spanish.pdf consulta 13/08/2018

Booth, Tony, y Ainscow, Mel (2011). Guía para la Educación Inclusiva: Desarrollando el aprendizaje y la participación en los centros escolares. Organización de Estados Iberoamericanos para la Educación, la Ciencia y la Cultura (OEI) y Fundación Benéfico-Social Hogar del Empleado (FUHEM). España.

Bromberg, Dinah. (2004). Los diez desafíos del diseño. Cuando falla la Victorinox.

Revista Portafolio. Volumen 1, No. 9. Venezuela. (Pp. 52-63).

Casanova, Ilya. (2011). Transversalidad y Desarollo de Competencias Profesionales. Tesis doctoral. Doctorado en Ciencias Humanas. Universidad del Zulia. Venezuela.

Duk, Cinthia, y Loren, Celina. (2010). Flexibilización del curriculum para atender la diversidad. Revista Rinace. Volumen 4, No. 1. España. (Pp. 187-210)

Feliz, Tiberio, y Ricoy, María. (2002). El diseño y desarrollo del currículum: Las adaptaciones curriculares. En González, Joaquín. Necesidades educativas especiales e intervención psicopedagógica. Universidad de Alcalá, Servicio de Publicaciones. España.

García, Mari. (2004). Desarrollo de niños ciegos y niños sordos: Análisis comparativo. Revista Seminario Médico. Volumen 56, No. 2. España. (Pp. 73-82).

García, Mirian. (2011). El niño con discapacidad visual.¿Cómo se desarrolla en el niño ciego la representación, interacción y función simbólica? Extraído de http://www.eduinnova.es/monografias2011/feb2011/visual.pdf consulta 04/09/2018.

Grau, Claudia, y Fernández, María. (2008). La atención a la diversidad y las adaptaciones curriculares en la normativa española. Revista Iberoamericana de Educación. No. 46/3. España. (Pp. 1-16)

Inciarte, Alicia y Canquiz, Liliana. (2008). Formación integral desde el enfoque por competencias. Maracaibo: Ediciones del Vice rectorado Académico. Universidad del Zulia.

Mace, Ronald. (1997). The Principles of Universal Design. Extraído de: https://www.ncsu.edu/ncsu/design/cud/about_ud/udprinciplestext.htm consulta 29/08/2017

Martín, José (1999). Adaptaciones Curriculares en E.S.O: Guía para cumplimentar el Documento Individualizado de Adaptación Curricular (DIAC). CIDE. España.

Olivares de Quintero, Ivonne. (2001). Un Modelo de Integración de las Funciones Universitarias Básicas. Extraído de: http://entretemas.com.ve/lineai/ArticulosAnteriores/IvonneQuinter/inde x.htm consulta 11/07/2018.

Organización de las Naciones Unidas (ONU). (2006). Convención sobre los derechos de las personas con discapacidad. Extraído de: 
http://www.un.org/esa/socdev/enable/documents/tccconvs.pdf, consulta $11 / 08 / 2017$

Organización Mundial de la Salud (OMS). (2001). Clasificación Internacional de Funcionamiento de la Discapacidad y de la Salud (CIF). Extraído de https://aspace.org/assets/uploads/publicaciones/e74e4-cif_2001.pdf, consulta 08/10/2017

Organización Nacional de Ciegos Españoles (ONCE). (2018). La discapacidad visual: características principales. Extraído de: https://www.once.es/dejanosayudarte/la-discapacidad-visual, consulta 26/08/2018

Padrón, José. (2002). Aspectos Básicos en la formación de investigadores Extraído de: http://padron.entretemas.com.ve/FormacInvest/AspBasicosFormInv.ht m, consulta 11/08/2018

Parra, Carlos. (2010). Educación inclusiva: Un modelo de educación para todos. Inclusión Social y Equidad en la Educación Superior (ISEES). España. (Pp. 73-84)

Rodríguez, Antonio, Rodríguez, Luis, y Gallego, José. (2012). La atención a la diversidad en el sistema educativo español. Revista Digital de Investigación Educativa. VI Edición. Año II. España. (Pp. 81-98).

Salvador, Francisco. (2001). Expresión escrita e disfuncións sensoriais. Revista Gallega do Ensino. Educación Especial, Número 32. España. (Pp. 309-330). Sánchez, Dalia. (2017). Estilos de Pensamiento y Discapacidad en el Contexto del Currículo para la Formación de Investigadores. Tesis doctoral. Doctorado en Ciencias Humanas. Universidad del Zulia. Venezuela. 